\title{
Inflammatory myofibroblastic tumour arising in the adrenal gland: a case report
}

\author{
My-Anh Tran-Dang ${ }^{1}$, Neal Banga ${ }^{2}$, Bernard $\mathrm{Khoo}^{3}$ and Alan WH Bates ${ }^{1 *}$
}

\begin{abstract}
Introduction: Inflammatory myofibroblastic tumour arising in the adrenal gland is exceptional. As far as we are aware, there have been only three previous reports in the literature. We report a fourth case.

Case presentation: A 29-year-old Caucasian man presented with upper quadrant pain due to a $15 \mathrm{~cm}$ heterogenous adrenal mass that displaced his liver. He underwent an open right adrenalectomy. Histopathological examination showed the mass to be an inflammatory myofibroblastic tumour, a histologically distinctive lesion composed of myofibroblasts, plasma cells, lymphocytes and histiocytes. Ten months later he is well with no sign of recurrence.
\end{abstract}

Conclusions: The lesion was indistinguishable on imaging from an adrenal cortical tumour. Surgical treatment is the same but inflammatory myofibroblastic tumour carries a favourable prognosis.

Keywords: Adrenal gland, Histology, Immunohistochemistry, Inflammatory myofibroblastic tumour

\section{Introduction}

Inflammatory myofibroblastic tumour (IMT) is an uncommon spindle-cell proliferation that occurs at various sites including the abdomen, lungs, orbit, gastrointestinal and genitourinary tracts, usually of children and young adults [1]; however, the overall age range and anatomical distribution is wide and IMTs may arise in almost any location. The adrenal gland is a rare primary site, with only three reports in the world literature [2-4]. We report a fourth case and describe the radiological and immunohistochemical features.

\section{Case presentation}

A 29-year-old Caucasian man presented with a 3- to 4-month history of discomfort in his right upper quadrant and right flank. He had no gastrointestinal or urinary disturbance and no endocrine symptoms. He had a previous medical history of latent tuberculosis, which was detected 3 months earlier, for which he was taking Rifanah (rifampicin and isoniazid).

On physical examination, there was anterior displacement of his liver by a palpable mass in the right hypochondrium. His blood pressure was normal. A dexamethasone suppression test, 24-hour urine catecholamine and metanephrine assays, and a plasma aldosterone: renin ratio were normal. An ultrasound scan of his abdomen and a computed tomography scan of his upper abdomen revealed a $15 \mathrm{~cm}$ heterogenous adrenal mass compressing his liver. These appearances raised the possibility of a primary adrenal carcinoma. A metaiodobenzylguanidine (MIBG) scan and single-photon emission computed tomography scan showed no significant uptake of tracer, making a phaeochromocytoma less likely. A fluorodeoxyglucose-positron emission tomography scan revealed a right adrenal mass of size $14.5 \times 9.5 \times 17.2 \mathrm{~cm}$ which was intensely metabolically active and showed dystrophic calcification and central necrosis, consistent with either adrenal cortical carcinoma or tuberculosis (Figure 1). His left adrenal gland was normal and there

\footnotetext{
* Correspondence: alan.bates@nhs.net

'Department of Cellular Pathology, Royal Free Hospital, London NW3 2QG, UK

Full list of author information is available at the end of the article
} 


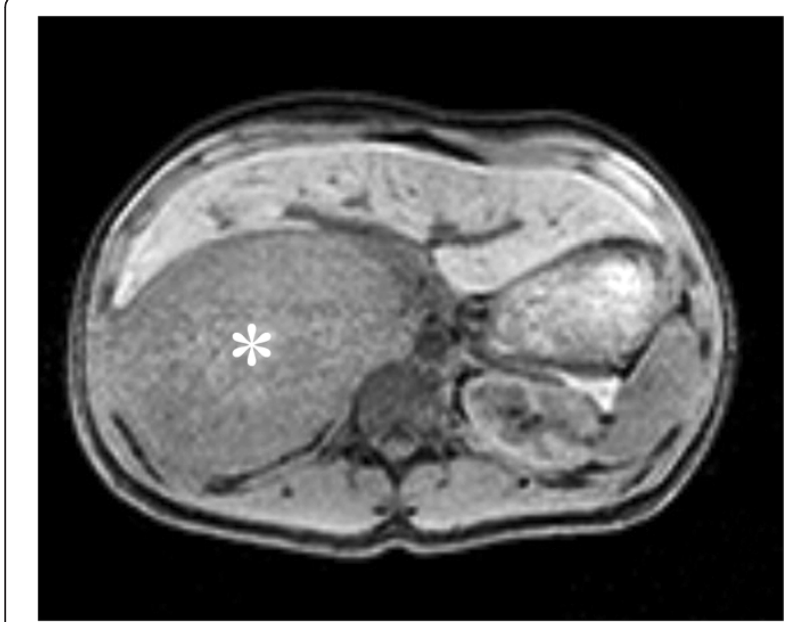

Figure 1 Positron emission tomography-computed tomography axial images showing an $18 \times 13 \times 9 \mathrm{~cm}$ well-defined heterogeneous hypodense lesion in the right adrenal gland (asterisk).

were no features to suggest active tuberculosis elsewhere. There was no evidence of metastatic disease. He underwent a right open adrenalectomy due to the large size of the mass. As predicted by the imaging, a massive right adrenal tumour was found adherent to his diaphragm and the posterior surface of his liver, but separate from his right kidney. It was possible to mobilize the tumour away from all of these structures without including them in the resection. The postoperative course was uneventful.

On gross examination, the resection specimen comprised a lobulated cream-coloured tumour weighing

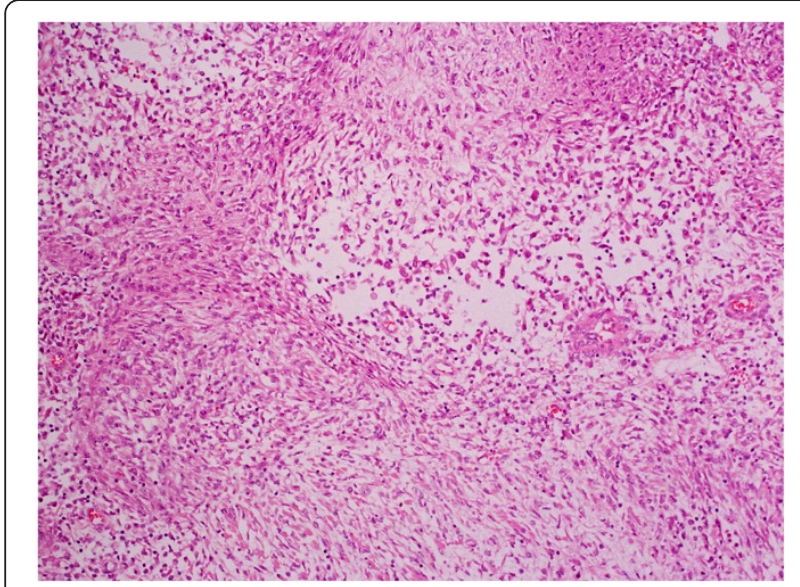

Figure 2 A low-power view of the tumour showing spindled and epithelioid cells arranged predominantly in a nondescript pattern with some areas showing a fascicular and focally whorled arrangement (haematoxylin and eosin).

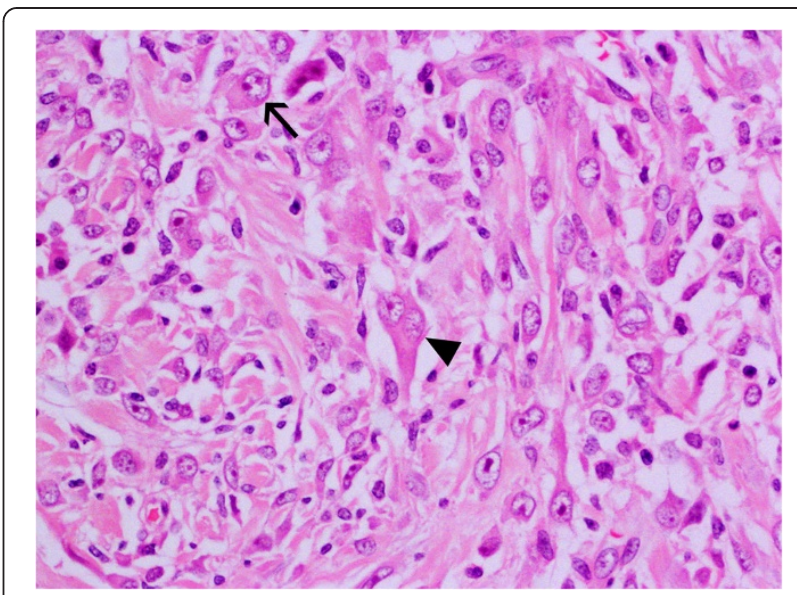

Figure 3 A high-power view showing plump spindle cells with mildly atypical nuclei. Occasional binucleated (arrowhead) and ganglion-like cells (arrow) are present.

$1.3 \mathrm{~kg}$ and measuring $18 \mathrm{~cm}$ in maximum dimension, with some compressed normal-looking adrenal gland at one end. The tumour had a smooth outer surface and a homogenous cut surface with a whorled appearance. There were occasional areas of haemorrhage. Microscopic examination showed a circumscribed tumour composed of plump, spindled cells and histiocytoid cells arranged haphazardly and in fascicles with intervening thick collagen bundles (Figure 2). There was an admixed moderate inflammatory cell infiltrate composed of lymphocytes, plasma cells and eosinophils. Occasional binucleated and ganglion-like cells were present (Figure 3). Mitoses were inconspicuous $(<1 / 30$ high-power fields) and no necrosis

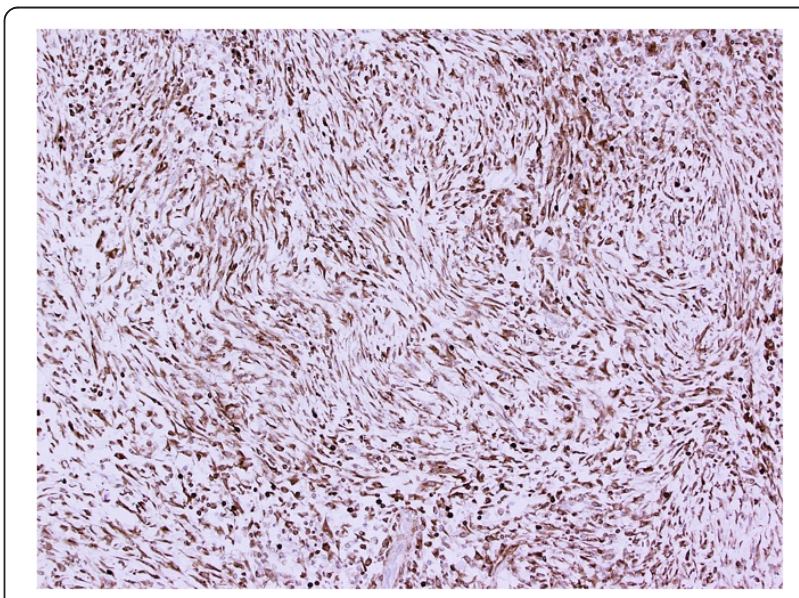

Figure 4 Immunohistochemical staining for bcl-2 shows diffuse strong positivity. 


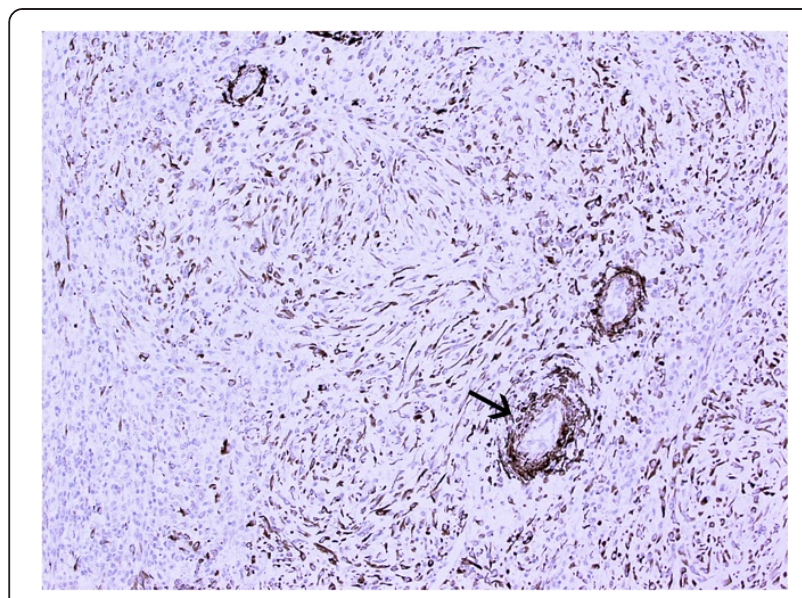

Figure 5 Focal positivity for smooth muscle actin, especially around small blood vessels (arrow).

was seen. On immunohistochemical examination, the tumour cells were strongly positive for bcl-2 with focal positivity for desmin, smooth muscle actin and beta-catenin (Figures 4 and 5). Tumour cells were negative for broad-spectrum cytokeratin, epithelial membrane antigen, S100, MelanA, CD34, CD117, DOG-1 and anaplastic lymphoma kinase (ALK)-1. MDM2 gene amplification analysis by fluorescent in situ hybridization was negative.

\section{Discussion}

The term 'inflammatory myofibroblastic tumour' was introduced in 1986 [5] to describe a lesion previously known as plasma cell granuloma [6] or inflammatory pseudotumour [7]. The advent of immunohistochemistry brought increasing awareness of its distinctive features and potential for local recurrence [8]. In recent years, cytogenetic and molecular genetic analysis has confirmed the neoplastic nature of these lesions [9].

On histological examination, IMTs are characterized by a proliferation of plump fibroblasts or myofibroblasts accompanied by a prominent infiltrate of chronic inflammatory cells, in particular plasma cells. This feature best distinguishes IMT from fibromatosis and fasciitis, with which it overlaps. A rare epithelioid variant of IMT is associated with an abdominal location and an aggressive clinical course [10].

On immunohistochemical examination, IMTs usually express actin and may also show staining for desmin and keratin. $A L K$ gene rearrangements, resulting in overexpression of ALK protein, are present in 30 to $40 \%$ of cases, mainly in the paediatric age group. Immunostaining for ALK is therefore seen only in a minority of cases. Of interest, the IMTs that have metastasized are most often ALK negative [11].

The three previous cases of adrenal IMT presented with flank pain or were incidental findings on abdominal imaging (Table 1). Clinical differential diagnoses include adrenocortical tumours, phaeochromocytoma, adrenal metastases and adrenal tuberculosis. Given that the imaging features of IMT are generally nonspecific, preoperative differentiation from other adrenal masses is very difficult. Complete surgical resection is therefore mandatory as malignancy cannot be excluded preoperatively. IMTs are usually asymptomatic, although 10 to $20 \%$ of cases are associated with pyrexia and weight loss. Tumour size at diagnosis is most often in the range of 5 to $10 \mathrm{~cm}$. The biological behaviour of IMT is indeterminate. After excision, 10 to $25 \%$ of patients have local recurrence. Less than $5 \%$ of cases have been reported to metastasize, but their behaviour is difficult to predict on morphological grounds, and it is increasingly believed that all cases of IMT are best regarded as low-grade sarcomas.

\section{Conclusions}

IMT in the adrenal gland is rare but must be considered in the differential diagnosis of radiologically suspicious adrenal masses. Recurrence or metastasis of adrenal IMT has not been reported but in view of the lesion's occasional metastatic potential at other sites careful postoperative follow up including repeat imaging at 6 months to exclude local recurrence is advised.

\section{Consent}

Written informed consent was obtained from the patient for publication of this case report and any accompanying images. A copy of the written consent is available for review by the Editor-in-Chief of this journal.

Table 1 Summary of reported cases of adrenal myofibroblastic tumour

\begin{tabular}{lccccc}
\hline Author (reference) & Patient age (years) & Sex & Presentation & Maximum tumour dimension (cm) & Recurrence \\
\hline Luo et al. [2] & Preschool & Female & Flank pain & ? & No \\
Wang et al. [3] & 57 & Female & Incidental & 8.5 & No \\
Chawla et al. [4] & 20 & Male & Flank pain & 7 & No \\
\hline
\end{tabular}




\section{Abbreviations}

ALK: Anaplastic lymphoma kinase; IMT: Inflammatory myofibroblastic tumour; MIBG: Metaiodobenzylguanidine.

\section{Competing interests}

The authors declare that they have no competing interests.

\section{Authors' contributions}

MT drafted the manuscript and performed histopathological and immunohistochemical analysis of the tumour. NB and BK provided the clinical information for the manuscript. AWHB revised the manuscript, approved the histopathological diagnosis of the tumour, and took the photomicrographs. All authors read and approved the final manuscript.

\section{Author details}

'Department of Cellular Pathology, Royal Free Hospital, London NW3 2QG, UK. ${ }^{2}$ Department of Endocrine Surgery, Royal Free Hospital, London NW3 2QG, UK. ${ }^{3}$ Department of Metabolism and Experimental Therapeutics, UCL Medical School, London NW3 2PF, UK.

Received: 12 June 2014 Accepted: 20 October 2014

Published: 7 December 2014

\section{References}

1. Fisher C: Soft tissue sarcomas with non-EWS translocations: molecular genetic features and pathologic and clinical correlations. Virchows Arch 2010, 456(2):153-166.

2. Luo LK, Shen HF, Zhou SY, Li JM, Xu WX: Inflammatory myofibroblastic tumour of adrenal. Zhonghua Bing Li Xue Za Zhi 2006, 35(4):252-253.

3. Wang T-Y, Chou J-W, Shih Y-S: Inflammatory myofibroblastic tumour mimicking adrenal incidentaloma. Inter Med 2011, 50:165-166.

4. Chawla A, Hameed Z, Mishra D, Monappa V: Adrenal inflammatory myofibroblastic tumour. BMJ Case Rep 2013, doi:10.1136/bcr-2013-010122.

5. Day DL, Sane S, Dehner LP: Inflammatory pseudotumor of the mesentery and small intestine. Pediatr Radiol 1986, 16(3):210-215.

6. Brunn $H$ : Two interesting benign lung tumours of contradictory histopathology: remarks on the necessity for maintaining the chest tumour registry. J Thorac Cardiovasc Surg 1939, 9:119-131.

7. Umiker WO, Iverson L: Postinflammatory 'tumours' of the lung: report of four cases simulating xanthoma, fibroma, or plasma cell tumour. J Thorac Cardiovasc Surg 1954, 28:55-63.

8. Pettinato G, Manivel JC, De Rosa N, Dehner LP: Inflammatory myofibroblastic tumor (plasma cell granuloma). Clinicopathologic study of 20 cases with immunohistochemical and ultrastructural observations. Am J Clin Pathol 1990, 94(5):538-546.

9. Sastre-Garau X, Couturier J, Derré J, Aurias A, Klijanienko J, Lagacé R: Inflammatory myofibroblastic tumour (inflammatory pseudotumour) of the breast. Clinicopathological and genetic analysis of a case with evidence for clonality. J Pathol 2002, 196(1):97-102.

10. Marino-Enriquez A, Wang WL, Roy A, Lopez-Terrada D, Lazar AJ, Fletcher CD, Coffin CM, Hornick JL: Epithelioid inflammatory myofibroblastic sarcoma; an aggressive intra-abdominal variant of inflammatory myofibroblastic tumour with nuclear membrane or perinuclear ALK. Am J Surg Pathol 2011, 35:135-144.

11. Coffin CM, Hornick JL, Fletcher CDM: Inflammatory myofibroblastic tumour; comparison of clinicopathologic, histologic, immunohistochemical features and ALK expression in atypical and aggressive cases. Am J Surg Pathol 2007, 31(4):509-520.

doi:10.1186/1752-1947-8-411

Cite this article as: Tran-Dang et al:: Inflammatory myofibroblastic tumour arising in the adrenal gland: a case report. Journal of Medical Case Reports 2014 8:411.

\section{Submit your next manuscript to BioMed Central and take full advantage of:}

- Convenient online submission

- Thorough peer review

- No space constraints or color figure charges

- Immediate publication on acceptance

- Inclusion in PubMed, CAS, Scopus and Google Scholar

- Research which is freely available for redistribution

Submit your manuscript at www.biomedcentral.com/submit 